\title{
Distribution Network Sensor Selection Based on Improved Membership Function
}

\author{
Erxia $\mathrm{Li}^{1}$, Yuling $\mathrm{Li}^{1}$, Naiqiang $\mathrm{Mao}^{2}$, Zhen $\mathrm{Wu}^{2}$ and Guoming $\mathrm{Ma}^{2}$ \\ ${ }^{1}$ China Electric Power Research Institute, Beijing \\ ${ }^{2}$ North China Electric Power University State Key Laboratory of Alternate Electrical Power System \\ with Renewable Energy Sources, Beijing
}

\begin{abstract}
Keywords: on-line monitoring; sensors; comprehensive evaluation; membership function
Abstract. The condition monitoring of distribution equipment is significant for safety and reliability of distribution network cooperation. In order to monitor the operating state of distribution equipment, first, we should learn about the function and characters of the different distribution and ensure the acquisition method to obtain the key characteristic quantity of distribution equipment. Then, we establish the system of comprehensive evaluation for the monitoring sensors application distribution equipment. Through the comprehensive evaluation of sensors, we can complete sensors selection and configuration and thus reduce the cost of monitoring system. To solve the ambiguity of sensors selection criteria, the author present two different comprehensive evaluation methods, one is aimed at applied object, other one is based on improved membership function.
\end{abstract}

\section{Introduction}

Distribution network is a complex, multilevel and distributed system which contains power transmission, transformation and use. With the development of industrial production, people pay more attention to safety and stability of power supply and the distribution equipment reliability have drawn extensive concern of the public and power experts. And the healthy condition of distribution equipment has significant influence on the system safety and stability of distribution network [1,2].

Gone with running time increase, insulation state of distribution equipment go worse and conventional preventive test can't discover the equipment defects without delay. The distribution network is in long distance and wide range, so the measurement and management for distribution equipment is complex and difficult. Hence the research about distribution equipment monitoring is significant to guarantee safe and reliable power supply.

In order to monitor the operation condition of distribution equipment, various sensors are used to sample the key characteristic quantity of equipment. But the sensors' selection and configuration need to consider and to save the equipment cost. In the economy evaluation system of distribution network management, some researchers adopt hierarchical thinking and fuzzy theory to establish appropriate assessment scheme. First, dividing the quality level and establishing weight coefficient is need. Then, by the membership function with the revised weight coefficient, it can obtain the score of evaluation project $[3,4]$.

\section{The Key Characteristic Quantity of Distribution Equipment}

Through the investigation of field operation experience and power company fault database, it can conclude the directed relationship between healthy condition and typical fault, such as equipment temperature, partial discharge, noise, vibration, leakage current and concentration of SF6.

2.1 On-line Monitoring of Transformer. The transformer is the key transmission facility in power supply. Its healthy condition directly influent the safety and reliability of whole distribution network[5]. About transformer condition monitoring, partial discharge, operation loss and oil temperature are primary monitoring variable and also are direct assessment basis[6].

Transformer on-line partial discharge monitoring is supported by advanced sensor technology and electronic technology. According to ultrasonic measurement theory, high frequency acoustic sensor 
which is installed on transformer shell can acquire the transient state acoustics signal. And electric monitoring method (shown in Fig. 1) is more sensitive compared with other methods.

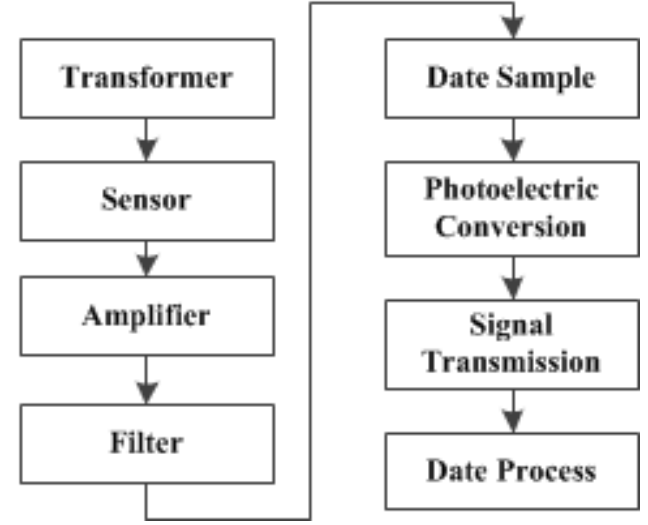

Fig.1 The flow chart of transformer partial discharge on-line monitoring

However, the research about transformer running loss isn't mature in technology. And field current change and load fluctuation is primary influencing factors which lead to measurement inaccuracy. And in most cases, it also is a disadvantage for measure load loss that sensor installation is inconvenient.

2.2 On-line Temperature Monitoring of Switch Cabinet. When the switch cabinet running, the contact temperature is primary basis which is used to evaluate the switch cabinet condition is good or bad[7]. Comparatively speaking, wireless temperature detection and fiber temperature detection is suit for the extensive use in the distribution equipment temperature measurement.

Fiber sensors' advantages are light mass, corrosion resistance and stand high temperature. And it also is anti-electromagnetic and low-attenuation. For the reason of considering facilities condition and cost, the important load facilities maybe need to install fiber sensors. And wireless temperature detection based on Zigbee is in low power consumption and convenient to network. So it is wildly used in local temperature measurement[8,9].

2.3 On-line Monitoring of Power Cable. Power cable is key facility in city distribution network, which undertake the mission of power transmission. For the monitoring of power cable, the temperature[10] and partial discharge of cable contact is chief monitoring characteristic quantity.

The insulation of power cable is closely related to contact partial discharge. The experts at home and abroad as well as some authority such as IEC, IEEE and CIGRE all think that partial discharge detection is an effective method for evaluating power cable insulation property. And distributed partial discharge detecting technology is a good choice[11].

2.4 Comprehensive Assessment for On-line Monitoring. The various sensors are wildly used in distribution network on-line condition monitoring. Different equipment need different sensors whose sensitivity, accuracy and cost is different. In order to control the monitoring system cost, it need to evaluate the economy and applicability of sensor, and at the same time meet the detecting requirements.

\section{The Sensor Comprehensive Evaluation Scheme Aimed at Demand}

Aimed at comprehensive assessment of sensor economy and applicability, it presents the assessment scheme need to consider the object level difference and divide different scale. For example, the areas which contain the hospital, chemical plant and important government agency are considered as important power supply nodes, and others as common nodes.

By the Eq.1 and Table.1, the cost of monitoring system can be evaluated.

$$
K=(\text { Cost offer }-A) / A
$$

Where

$\mathrm{K}$ The error of cost offer

A Cost standard 
Table 1 the error of cost offer

\begin{tabular}{|c|c|c|c|c|c|c|c|c|}
\hline $\mathrm{K}$ & $\begin{array}{c}\text { Above } \\
+15 \%\end{array}$ & $+10 \%$ & $+5 \%$ & 0 & $\begin{array}{c}-4 \sim \\
-5 \%\end{array}$ & $-8 \%$ & $-10 \%$ & $\begin{array}{c}\text { Above } \\
-15 \%\end{array}$ \\
\hline score & 35 & 40 & 55 & 65 & 70 & 68 & 65 & 58 \\
\hline
\end{tabular}

For the evaluation and selection of sensors, the evaluated standard that is set for stability, sensitivity, accuracy, lifetime and cost is different in different scale node. For example, in an important node, the stability and sensitivity is considered seriously, but the sensor cost is not so important.

According to characteristic quantity and detected demand, the appropriate sensors that meet the demand of system measurement would be choose quickly and in economy the cost is low.

\section{The Sensor Comprehensive Evaluation Scheme Based on Improved Membership Function}

Compared with the sensor comprehensive evaluation scheme aimed at demand, the evaluation scheme which is based on improved membership function adopt fuzzy theory to build membership function. Considering about membership function, it quantify every index grade and build calculation model[12].

4.1 The Grading and Weight Definition. It presents the reference standard of sensor index grading (shown in Table 2). The reference standard contains index error, grade and index score. The L1 is excellent and the L5 is bad.

Table 2 The index and grading standards of sensors

\begin{tabular}{|c|c|c|c|c|c|c|}
\hline Grade & $\begin{array}{c}\text { Stability } \\
\text { Error (\%) }\end{array}$ & $\begin{array}{c}\text { Sensitivit } \\
\text { y Error } \\
(\%)\end{array}$ & $\begin{array}{c}\text { Accuracy } \\
\text { Error (\%) }\end{array}$ & $\begin{array}{c}\text { Cost Error } \\
(\%)\end{array}$ & $\begin{array}{c}\text { Lifetime } \\
\text { Error (\%) }\end{array}$ & $\begin{array}{c}\text { Applicabilit } \\
\text { y Error (\%) }\end{array}$ \\
\hline L1 & $0 \sim 1$ & $0 \sim 0.5$ & $0 \sim 2$ & $0 \sim 15$ & $0 \sim 15$ & $0 \sim 10$ \\
\hline L2 & $1 \sim 2$ & $0.5 \sim 1$ & $2 \sim 5.5$ & $15 \sim 30$ & $15 \sim 30$ & $10 \sim 25$ \\
\hline L3 & $2 \sim 3$ & $1 \sim 1.5$ & $5.5 \sim 7.5$ & $30 \sim 40$ & $30 \sim 40$ & $25 \sim 35$ \\
\hline L4 & $3 \sim 4$ & $1.5 \sim 2$ & $7.5 \sim 10$ & $40 \sim 50$ & $40 \sim 50$ & $35 \sim 45$ \\
\hline L5 & $>4$ & $>2$ & $>10$ & $>50$ & $>50$ & $>45$ \\
\hline
\end{tabular}

The traditional Analytic Hierarchy Process (AHP) would compare one index and another index $n(n-1) / 2$ times to establish weight coefficient of every index and the process is complex. But the improved AHP just need to compare index $(n-1)$ times, and can obtain the judgment matrix $\left(R_{n \times n}\right)$. Hypothetically, $w_{i}$ is the subjective weight of ith index, and $r_{i j}$ is the degrees of importance of ith index relative to jth index. The Eq.1 can be used to calculate the subjective weight coefficient.

$$
\begin{gathered}
\omega_{i}=\sqrt[n]{\prod_{j=1}^{n} r_{i j}} / \sum_{i=1}^{n} \sqrt[n]{\prod_{j=1}^{n} r_{i j}} \\
(i=1,2 \cdots, n ; j=1,2 \cdots, n)
\end{gathered}
$$

The Entropy Method is an objective method which also is applied to calculate the weight coefficient according to the size of index value information. The matrix ${ }_{D_{n \times m}}$ indicate that the number of index is $n$ and the dimension of data is $m$. Transformed into per-unit values, $S=\left(s_{i j}\right)_{n \times m}$, where $s_{i j}=\frac{\max d_{i j}-d_{i j}}{\max d_{i j}-\min d_{i j}}$, and $d_{i j}$ is the jth measurement value of ith index. The ith index entropy value is

$$
H_{i}=-(\ln m)^{-1} \sum_{j=1}^{m} p_{i j} \ln p_{i j}, i=1,2 \cdots, n, p_{i j}=s_{i j} / \sum_{j=1}^{m} s_{i j}
$$

If the objective weight coefficient of ith index is $v_{i}$,so

$$
W_{i}=\frac{\omega_{i} v_{i}}{\sum_{i=1}^{n} \omega_{i} v_{i}}, i=1,2 \cdots, n
$$


The total weight coefficient include subjective weight coefficient and objective weight coefficient, and is $W=\left[W_{1}, W_{2} \cdots, W_{n}\right]$. Then,

$$
W_{i}=\frac{\omega_{i} v_{i}}{\sum_{i=1}^{n} \omega_{i} v_{i}}, i=1,2 \cdots, n
$$

4.2 Improved Membership Function Model. The fuzziness and the uncertainty of sensor index can transform each other[13]. The membership of quality class (shows in Table 2) is equal to fuzziness. Shown in Fig.2, L1 L5 is five different quality classes and the fuzziness of between two different quality classes is quantitative. The values of $X_{1}, X_{2}$ depend on the actual situation.

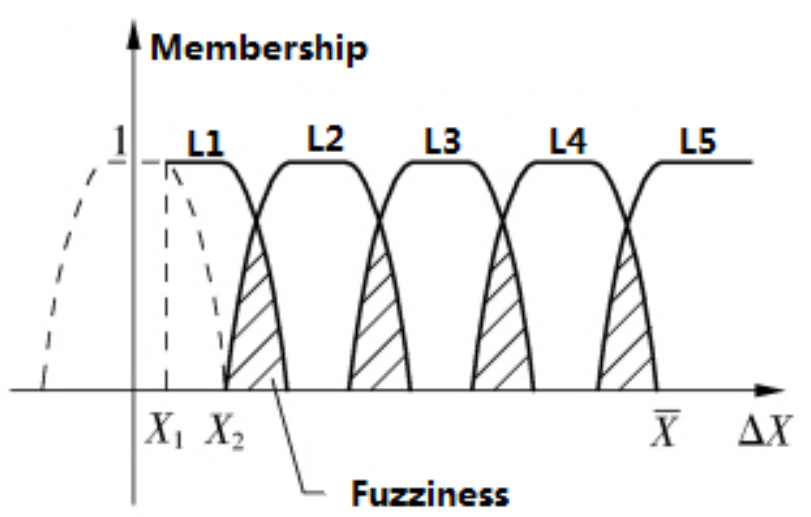

Fig.2 The membership function of evaluation index relative to each grade

First, the membership function of the $\mathrm{L} 1$ (excellent class) is

$\mu(\Delta X)= \begin{cases}1, & 0 \leq \Delta X \leq X_{1}+C \\ \frac{1}{2}-\frac{1}{2} \sin \varphi, & X_{1}+C<\Delta X<X_{2}+C \\ 0, & \Delta X \geq X_{2}+C\end{cases}$

Where $\varphi=\frac{\pi}{X_{2}-X_{1}}\left(\Delta X-\frac{X_{1}+X_{2}}{2}\right), C$ is a constant, in general, $C=\frac{1}{8} \bar{X}$.

Then, the membership function of the L1 L3 is

$$
\mu(\Delta X)=\left\{\begin{array}{lc}
0, & \Delta X \leq-X_{2}+(C+n k) \\
\frac{1}{2}+\frac{1}{2} \sin \varphi, & -X_{2}+(C+n k)<\Delta X<-X_{1}+(C+n k) \\
1, & -X_{1}+(C+n k) \leq \Delta X \leq X_{1}+(C+n k) \\
\frac{1}{2}-\frac{1}{2} \sin \varphi, & X_{1}+(C+n k) \leq \Delta X \leq X_{2}+(C+n k) \\
0, & X_{2}+(C+n k) \leq \Delta X
\end{array}\right.
$$

Where $k=\frac{1}{4} \bar{X}$, and $n=1,2,3$.

Last, the membership function of the L5 (bad class) is

$$
\mu(\Delta X)= \begin{cases}1, & \Delta X \geq-X_{1}+(C+n k) \\ \frac{1}{2}+\frac{1}{2} \sin \varphi, & -X_{2}+(C+n k)<\Delta X<-X_{1}+(C+n k) \\ 0, & -X_{2}+(C+n k) \leq \Delta X\end{cases}
$$

Where $n=4$.

The mathematic model based on membership function can solve the fuzziness between correlative quality class and correlative index, and at the same time, it builds the tight relation between correlative quality classes.

4.3 The Algorithm Design. The process of the sensor comprehensive evaluation scheme is follow: 
(1) Transform the sensor index value into per-unit value and obtain the standard data which can be used directly to evaluate the sensor performance.

(2) Apply the Eq.1,Eq.3 and Eq.4 to calculate the subjective weight, objective weight and total weight of per index.

(3) Apply Eq.5 Eq.7 to fuzzy up sensor index quality class and obtain the membership matrix $u$.

(4) Apply the Eq.8 to evaluate the membership relationship between total condition and other quality class fuzzy subset.

$B=W * u$

The class score of $\mathrm{L} 1 \sim \mathrm{L} 5$ is $c_{1}, c_{2}, c_{3}, c_{4}, c_{5}$, and the space of score is same. Finally it can obtain the evaluation factor $\sum_{j=1}^{m} b_{j} c_{j}$ on the evaluation point and the calculation equation is

$$
f_{P Q}=\sum_{j=1}^{m} b_{j} c_{j} / \sum_{j=1}^{m} b_{j}
$$

Where $b_{j}$ is the membership which is related to jth quality class.

\section{Case Analysis}

Now, there are three kinds sensors (A, B and C) that can be selected for power company. Considering the accuracy, reliability, lifetime and cost, which sensor is best one suit for procurement? The index and parameter value of three kinds is shown in Table 3.

Table 3 sensor parameter values

\begin{tabular}{|c|c|c|c|}
\hline Sensor & A & B & C \\
\hline Cost (¥) & 200 & 260 & 300 \\
\hline Lifetime (Year) & 20 & 25 & 30 \\
\hline Reliability & 35 & 40 & 45 \\
\hline Accuracy & 0.5 & 0.4 & 0.2 \\
\hline
\end{tabular}

\subsection{Index Weight Calculation.}

(1) Calculate subjective weight coefficient. For certain index, important scale: cost $>$ lifetime $>$ reliability > accuracy, so according to the important scale, it can assume the expected value: $R_{12}$ =1.3(Reliability $>$ Accuracy), $\quad R_{23} \quad=1.3$ (Lifetime $>$ Reliability), $\quad R_{34} \quad=3.63$ (Cost $>>$ Lifetime). According to the consistency of matrix ( $\forall k \quad R_{i j}=R_{i k} * R_{k j}$ ), judgment matrix is follow:

$$
R=\left[\begin{array}{cccc}
1 & 1.3 & 1.69 & 6.135 \\
\frac{1}{1.3} & 1 & 1.3 & 4.179 \\
\frac{1}{1.69} & \frac{1}{1.3} & 1 & 3.63 \\
\frac{1}{6.135} & \frac{1}{4.179} & \frac{1}{3.63} & 1
\end{array}\right]
$$

According to equation $\omega_{i}=\sqrt[n]{\prod_{j=1}^{n} r_{i j}} / \sum_{i=1}^{n} \sqrt[n]{\prod_{j=1}^{n} r_{i j}}$, the subjective weight coefficient can be calculated: $(i=1,2 \cdots, n ; j=1,2 \cdots, n)$

$\omega=\left[\begin{array}{llll}0.067 & 0.236 & 0.298 & 0.399\end{array}\right]$.

(2) Calculate objective weight coefficient. Apply the equation $s_{i j}=\frac{d_{i j}}{\max d_{i j}-\min _{i j}}$ to obtain the per-unit value of index data and the standard data matrix is follow: 


$$
S=\left[\begin{array}{lll}
5 / 3 & 4 / 3 & 2 / 3 \\
3.5 & 4 & 4.5 \\
2 & 2.5 & 3 \\
2 & 2.6 & 3
\end{array}\right]
$$

By calculation, $H=\left[\begin{array}{llll}0.987 & 0.988 & 0.995 & 0.943\end{array}\right]$, so the result of the objective weight coefficient is $v=\left[\begin{array}{llll}0.15 & 0.14 & 0.06 & 0.66\end{array}\right]$, and the total weight coefficient is $W=\left[\begin{array}{llll}0.031 & 0.102 & 0.055 & 0.812\end{array}\right]$.

5.2 The formation of index membership matrix. The reference value of $X_{1}, X_{2}$ of index is shown in Table 4.

Table 4 The reference of $X_{1}, X_{2}$
\begin{tabular}{|c|c|c|c|}
\hline & $\mathrm{X}_{1}$ & $\mathrm{X}_{2}$ & $\bar{X}$ \\
\hline Cost (¥) & 1.5 & 2 & 3.5 \\
\hline Lifetime(Year) & 2.8 & 2.2 & 1.8 \\
\hline Reliability & 4 & 3.4 & 3 \\
\hline Accuracy & 0.5 & 0.8 & 2 \\
\hline
\end{tabular}

Apply Eq.5 Eq.7 to calculate the membership matrix of sensor A, B and C. The matrix is follow:

$$
\begin{gathered}
\mu_{A}=\left[\begin{array}{lllll}
0 & 0 & 0 & 1 & 0 \\
0 & 1 & 0 & 0 & 0 \\
0 & 0 & 0 & 1 & 0 \\
0 & 1 & 0 & 0 & 0
\end{array}\right] \\
\mu_{B}=\left[\begin{array}{lllll}
0 & 0 & 0 & 1 & 0 \\
0 & 1 & 0 & 0 & 0 \\
0 & 0 & 1 & 0 & 0 \\
0 & 0 & 0 & 1 & 0
\end{array}\right] \\
\mu_{C}=\left[\begin{array}{ccccc}
0 & 0.29 & 0.71 & 0 & 0 \\
1 & 0 & 0 & 0 & 0 \\
1 & 0 & 0 & 0 & 0 \\
0 & 0 & 1 & 0 & 0
\end{array}\right]
\end{gathered}
$$

5.3 The Membership Extent of Fuzzy Subset. By the equation $B=W^{*} u$, it obtain the membership extent matrix:

$$
\begin{aligned}
B_{A} & =\left[\begin{array}{lllll}
0 & 0.9140 & 0 & 0.0860 & 0
\end{array}\right] \\
B_{B} & =\left[\begin{array}{lllll}
0 & 0.1020 & 0.0550 & 0.8430 & 0
\end{array}\right] \\
B_{C} & =\left[\begin{array}{lllll}
0.1570 & 0.0090 & 0.8340 & 0 & 0
\end{array}\right]
\end{aligned}
$$

If the scores of $\mathrm{L} 1 \sim \mathrm{L} 5$ level are $c_{1}=5, c_{2}=4, c_{3}=3, c_{4}=2, c_{5}=1$, and the score is in the order from good to bad. By the weighted average method, it can calculate the evaluation factor $\sum_{j=1}^{m} b_{j} c_{j}$. Finally, the comprehensive evaluation result of the three kinds of sensor is shown in Table 5.

Table 5 The evaluation result of the sensor

\begin{tabular}{|c|c|}
\hline Sensor & Evaluation Result \\
\hline A & 3.828 \\
\hline B & 2.259 \\
\hline C & 3.323 \\
\hline
\end{tabular}

The evaluation result of sensor suggests, the sensor A is the best choice for engineer application. 


\section{Conclusion}

For the selection of distribution monitoring sensor, it presents two sensor comprehensive evaluation schemes. And one scheme is aimed at object demand, and other one is based on improved membership function.

Compared with each other, the former is focused on application convenience of scheme, but the accuracy requirement is low. And the latter emphasizes evaluation accuracy. It builds the calculation model based on the membership function to evaluate the object objectively, but the algorithm is complex. In actually, the scheme is selected depended on the user demand.

\section{References}

[1] Hua Zhao, Zhuiding Wang, Kaigui Xie, Wenyuan Li. Comparative Study on Reliability Assessment Methods for Medium Voltage Distribution Network[J]. Power System Technology, 2013,11:3295-3302.

[2] Mingjun Yuan, Studies on Distribution Systrm Reliability Assessment Method and Applicatuon[D]. Shandong University,2011

[3] Liye Ma, Zhigang Lu, Huawei Hu. A Fuzzy Comprehensive Evaluation Method for Economic Operation of Urban Distribution Network Based on Interval Number[J]. Transactions of China Electrotechnical Society. 2012,08:163-171.

[4] Fan Wang. The Economic Evaluation of the Distribution System Based on Multi-evaluation model[D].Yanshan University,2010.

[5] Hua Huang, Linghui Yang, Long Li. Evaluation and Selection of On-line Monitoring System for Transmission and Distribution Equipment.[J]East China Electric Power, ,2006,08:40-42.

[6] Ningning Zhan. Research and Application of Transformer Online Monitoring Sensor [D]. North China Electric Power University,2014

[7] Xiaohua Wang, Bingrong Su, Mingzhe Rong. Development of On-line Monitoring System Voltage Switchgear [J]. High Voltage Apparatus, 2009,03:52-55.

[8] Yi Tian, Xinbo Huang. On-line Monitoring System of Temperature Inside HV Switchgear Cabinet [J]. High Voltage Apparatus, 2010,03:64-67+71.

[9] Xuewei Chen, Guoming Ma, Chengrong Li. Wireless Temperature Measuring System Design for High Voltage Switchgear and Its Reliability Research [J] High Voltage Apparatus, 2015,06:152-160.

[10] Chao Peng, Jiankang Zhao, Fugui Miao. Distribution Temperature System Applied in Cable Temperature Measurement [J]. High Voltage Engineering, 2006,08:43-45.

[11] Yun Jiang, Yunjie Zhou. Application of Distributed Partial Discharge On-line Monitoring Technology in Shanghai $500 \mathrm{kV}$ XLPE Power Cable Line[J]. High Voltage Engineering, 2015,04:1249-1256.

[12] Zhong Wan, Wendong Liang, Zongjuan Lu. Study on the Membership Interval Distribution Function for Fuzzy Sets[J]. Journa l of Chongqing University of Techno logy( Natural Science), 2011,01:107-112

[13] Kaidi Liu, Yanjun Pang, Wenguo Li. Membership Transforming Algorithm inMulti-index Decision and Its Application[J]. Acta Automatica Sinica , 2009,03:315-319. 